\title{
ON A POSITIVE TRIGONOMETRIC SUM
}

\section{RICHARD ASKEY, ${ }^{1}$ JAMES FITCH AND GEORGE GASPER ${ }^{2}$}

We give a new proof of the following theorem of P. Turán [2]. See [1] for another proof, shorter than Turán's but longer than this proof.

THEOREM. Let $\sum_{n=1}^{N} a_{n} \sin (2 n-1) \theta \geqq 0,0 \leqq \theta \leqq \pi$. Then

$$
\sum_{n=1}^{N} \frac{a_{n} \sin n \phi}{n}>0, \quad 0<\phi<\pi,
$$

unless all $a_{n}=0$.

A simple computation shows that

$$
\frac{d}{d y} \frac{\sin \alpha y}{\alpha(\sin y)^{\alpha}}=-\frac{\sin (\alpha-1) y}{(\sin y)^{\alpha+1}} .
$$

Letting $\alpha=2 n$ and $y=\phi / 2$ we see that

$$
\frac{\sin n \phi}{n}=2 \int_{\phi / 2}^{\pi / 2}\left(\frac{\sin \phi / 2}{\sin \theta}\right)^{2 n} \frac{\sin (2 n-1) \theta}{\sin \theta} d \theta .
$$

Thus

$$
\sum_{n=1}^{N} \frac{a_{n} \sin n \phi}{n}=2 \int_{\phi / 2}^{\pi / 2} \sum_{n=1}^{N} a_{n}\left(\frac{\sin \phi / 2}{\sin \theta}\right)^{2 n} \sin (2 n-1) \theta \frac{d \theta}{\sin \theta} .
$$

But $\sum_{n=1}^{N} a_{n} r^{2 n-1} \sin (2 n-1) \theta>0,0<r<1$, if $\sum_{n=1}^{N} a_{n} \sin (2 n-1) \theta \geqq 0$ and not all $a_{n}$ are zero and this completes the proof.

\section{REFERENCES}

1. C. Hyltén-Cavallius, $A$ positive trigonometrical kernel, Tolfte Skand. Mathematiker kongressen 1953, Lund (1954).

2. P. Turán, On a trigonometric sum, Ann. Polon. Math. 25 (1953), 155-161.

UNIVERSITY OF WISCONSIN

Received by the editors February 26, 1968.

1 Sponsored by the Mathematics Research Center, United States Army, Madison, IVisconsin, under Contract No. DA-31-124-ARO-D-462.

2 Supported in part by National Science Foundation grant GP-6764. 